\title{
Abbreviations and conventions used in the text
}

$/ \ldots /$ enclose a phonemic transcription

[...] enclose a phonetic transcription, where the actual sounds made are the focus of attention

$<\ldots>$ enclose an orthographic representation; enclose URLs

FORCE small capitals indicate lexical sets, see section 6.1

* not a grammatical sentence/construction

Aus Australia(n)

CDN Canada/Canadian

GA General American, see section 6.1

NAm North American

NZ New Zealand

RP Received Pronunciation, see section 1.1

SA South Africa(n)

Transcription systems for RP and GA are those used in the companion volume, McMahon (2002). 